\title{
Cartography of the magnetic fields and chemical spots of Ap stars
}

\section{James Silvester ${ }^{1,2}$, O. Kochukhov ${ }^{3}$, G. A. Wade ${ }^{1}$, N. Piskunov ${ }^{3}$,} J. D. Landstreet ${ }^{4}$ and S. Bagnulo ${ }^{5}$

${ }^{1}$ Department of Physics, Royal Military College of Canada, Kingston, ON, Canada K7K 4B4

${ }^{2}$ Department of Physics, Queen's University, Kingston, Ontario, Canada

${ }^{3}$ Department of Physics and Astronomy, Uppsala University, Box 515, 75120 Uppsala, Sweden

${ }^{4}$ Dept. of Physics \& Astronomy, Univ. of Western Ontario, London, ON, Canada, N6A 3K7

${ }^{5}$ Armagh Observatory, College Hill, Armagh BT61 9DG, Northern Ireland

\begin{abstract}
We will introduce a project using Magnetic Doppler Imaging (MDI) to create assumption-free vector magnetic field maps and chemical surface structure maps of chemically peculiar A and B type (or Ap) stars. We are exploiting the latest generation of spectropolarimeters (NARVAL at the Pic du Midi observatory, and ESPaDOnS at the Canada-France-Hawaii telescope), to obtain high-resolution time series of Stokes IQUV spectra of a selection of Ap stars. The spectra have superior signal-to-noise ratio, resolution and wavelength coverage to those used previously. This combined with the ground-breaking inversion techniques introduced by Kochukhov et al. (2002) results in maps which represent the state-of-the-art in the field of stellar cartography. These maps will allow us to better understand the links between the magnetic field and the physical processes leading to the formation of chemical structures in the photosphere and allow us to address questions surrounding the detailed magnetic field geometry of Ap stars.
\end{abstract}

Keywords. Stars: magnetic fields - techniques: polarimetric - stars: early-type - stars: chemically peculiar

\section{Introduction}

Magnetic fields play a fundamental role in the physics of the atmospheres of a significant fraction of stars on the H-R diagram. In early-type stars, the large-scale surface magnetic field is static on timescales of at least many decades, and appears to be "frozen" into a rigidly rotating atmosphere. The magnetic field is globally organised, permeating the entire stellar surface, with a relatively high field strength. The presence of the magnetic field strongly influences energy and mass transport (e.g., diffusion, convection and weak stellar winds) within the atmosphere of a star, and results in the presence of strong chemical abundance nonuniformities in photospheric layers, although to what level the magnetic field plays a role in these processes is still unclear.

The very first measurements of rotationally-modulated Zeeman circular and linear polarisation resolved within stellar line profiles were reported by Wade et al. (2000). This data set was later used with the new Magnetic Doppler Imaging technique (MDI), described by Piskunov \& Kochukhov (2002) and Kochukhov \& Piskunov (2002), to construct the very first assumption-free, high resolution maps of the surface vector magnetic field of the Ap stars 53 Cam (Kochukhov et al. 2004) and $\alpha^{2}$ CVn (Kochukhov et al. in prep). The unique maps of $53 \mathrm{Cam}$ and $\alpha^{2} \mathrm{CVn}$ reveal that the detailed magnetic topology departs significantly from the commonly-assumed low-order multipolar geometry, and that the abundances were not distributed uniformly with respect to the magnetic 


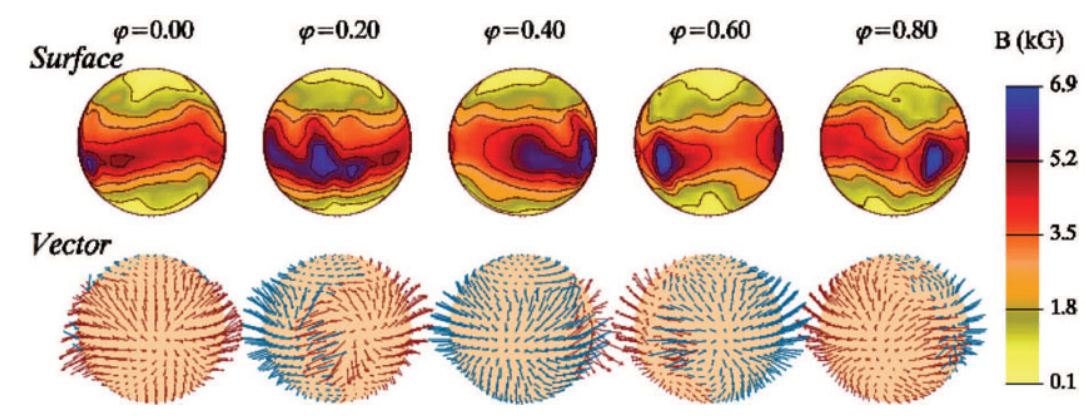

Figure 1. Preliminary magnetic field orientation and intensity maps of $49 \mathrm{Cam}$, as recovered using the MDI technique and the new IQUV spectropolarimetric data.

field, but localised in complex structures. The data used in these early maps represented the best data set obtained from several years of MuSiCoS observations. However, with this data set the Stokes $Q$ and $U$ signatures were only really detectable in 3 strong lines, with a $\mathrm{S} / \mathrm{N}$ of 5 or less in a sample of 2 or 3 stars. Due to the low signal-to-noise ratio and resolving power achievable with the MuSiCoS instrument, only an extremely limited range of stellar properties (rotation, mass, temperature, magnetic field, etc.) which may influence the phenomena of interest could be studied using the MuSiCoS data.

\section{The Project}

With the vastly improved data now available with ESPaDOnS and NARVAL we are creating the new generation of MDI maps using the INVERS10 inversion code, with these maps we will be able to characterize the magnetic field geometry of Ap stars, further refine the current model of the magnetic field structure and characterize the abundance surface structure. To test the quality of this new data and to confirm consistency with previous studies, the longitudinal field and net linear polarisation observed in 49 Cam were compared with measurements obtained by Wade et al. (2000). The data proved to be consistent with the previous MuSiCoS measurements, with uncertainties on average 3 to 4 times smaller than those obtained with MuSiCoS. A preliminary surface and vector field map for 49 Cam constructed using ESPaDOnS and NARVAL observations and using INVERS10 is shown in Fig. 1.

Once complete phase coverage is obtained for 49 Cam, HD 32633 and $\alpha^{2}$ CVn, the next generation of MDI maps can be produced. This will be followed by a multipolar analysis on the resulting magnetic maps to determine the true magnetic field structure of each star and an investigation into any relationships between the magnetic field and abundance structures by performing a pixel by pixel analysis.

\section{References}

Kochukhov, O., Bagnulo, S., Wade, G. A., Sangalli, L., Piskunov, N., Landstreet, J. D., Petit, P., \& Sigut, T. A. A. 2004, A\&A 414, 613

Kochukhov O., Piskunov N., Ilyin I., Ilyina S., \& Tuominen I. 2002, A\&A 389, 420

Kochukhov O. \& Piskunov N. 2002, A\&A 288, 868

Kochukhov, O. \& Bagnulo, S. 2006, A\&A 450, 763

Piskunov N. \& Kochukhov O. 2002, A\& $A$ 381, 736

Wade G. A., Donati J.-F., Landstreet J. D., \& Shorlin S. L. S. 2000b, MNRAS 313, 823 